\title{
Competitive evaporation in arrays of droplets
}

\author{
A. M. Lacasta \\ Departament de Física Aplicada, Universitat Politècnica de Catalunya, Avinguda Dr. Gregorio Marañon 50, E-08028 Barcelona, Spain \\ I. M. Sokolov \\ Theoretishe Polymerphysik, Universität Freiburg, Rheinstraße 12, D-79104 Freiburg i. Br., Germany \\ J. M. Sancho \\ Institute for Nonlinear Science, Department 0407, University of California, San Diego, 9500 Gilman Drive, \\ La Jolla, California 92093-0407 \\ and Departament d'Estructura i Constituents de la Matèria, Universitat de Barcelona, Avinguda Diagonal 647, \\ E-08028 Barcelona, Spain \\ F. Sagués \\ Departament de Química Física, Universitat Barcelona, Avenida Diagonal 647, E-08028 Barcelona, Spain
}

(Received 30 September 1997)

\begin{abstract}
We consider the evaporation of periodic arrays of initially equal droplets in two-dimensional systems with open (absorbing) boundaries. Our study is based on the numerical solution of the Cahn-Hilliard equation. We show that due to cooperative effects the droplets which are further from the boundary may evaporate earlier than those in the boundary's vicinity. The time evolution of the overall amount of matter in the system is also studied. [S1063-651X(98)03705-2]
\end{abstract}

PACS number(s): 83.70.Hq, 64.70.Fx, 05.70.Ln

The kinetics of first-order phase transitions has drawn much attention during the whole last century, giving a vivid example of pattern formation in systems attempting to spontaneously reach their equilibrium state [1-8]. The typical physical situations here are the liquid-gas systems, the binary fluid mixtures, or binary alloys. The systems quenched from the disordered (homogeneous) state into a domain of parameters where this homogeneous state is unstable and then relaxing to equilibrium (corresponding to full phase separation) show strongly nonlinear, cooperative behavior. In its late stages this process can be described by Lifshitz-Slyosov theory [3], describing the formation of clusters (droplets) and the redistribution of matter between them so that the larger droplets grow at the cost of smaller ones, which are thermodynamically less stable due to their higher surface energy.

The description of phase separation processes normally supposes the system to be closed, so that the overall amount of matter (the integral of the order parameter over the system's volume) is conserved. In the present article we consider a different situation, when open boundaries of the system lead to a decrease in the overall amount of matter. The simplest experimental geometry of the system corresponds to a flat cuvette containing droplets and open from one side to a large vessel with saturated vapor. In this geometry the different droplets evaporate not independently from each other (as it could be if the upper cover plate was absent) but as a cooperative process due to their strong interaction via exchanging matter through the gas phase. We confine ourselves to initially periodic arrangements of droplets and consider the change of their structure in time and the overall kinetics of evaporation. The most striking (and rather unexpected) qualitative effect found here is the possible reversion of the order of evaporation of subsequent droplets, so that some of the droplets which are nearer to the open boundary evaporate later than the more distant ones (see Fig. 1). This qualitative effect is probably amenable to experimental observation.

We turn now to the theoretical discussion of the situation. The phenomenological description of systems showing domain growth and phase separation often starts from timedependent Ginzburg-Landau models with conserved order
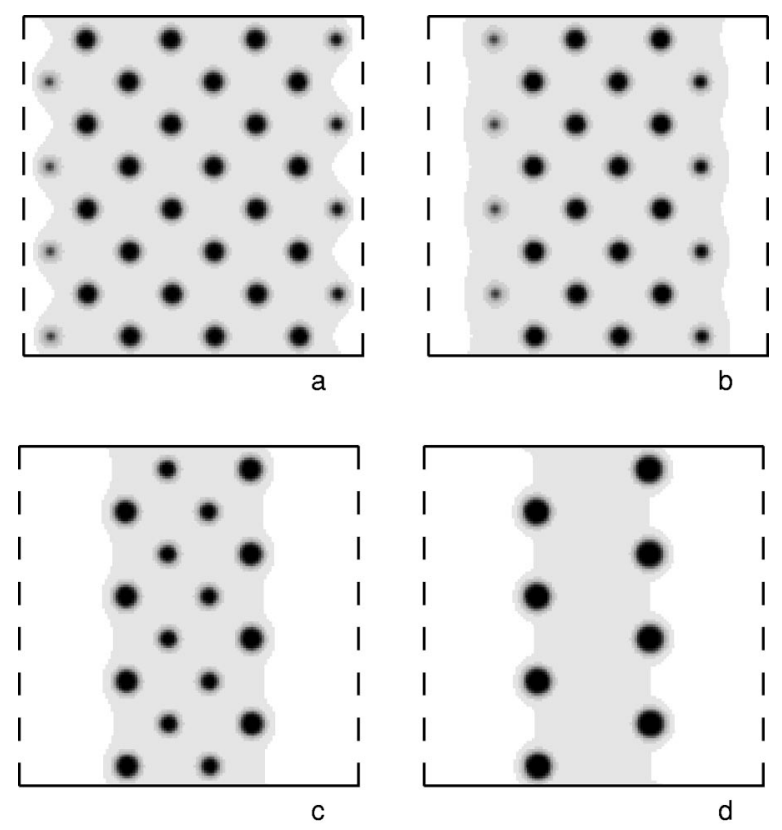

FIG. 1. Patterns of evaporation of a two-dimensional periodic array of droplets of initial radius $R=5$ (see text for details). The gray scale indicates the local concentration $c(r, t)$. The values of time are (a) $t=250$, (b) $t=1000$, (c) $t=2000$, and (d) $t=3000$. 
parameter. The simplest model of this type, neglecting all the macroscopic motions and taking into account only the diffusive redistribution of matter, is model $\mathrm{B}$ of critical dynamics and is described by the Cahn-Hilliard equation $[4,6]$. Its dimensionless form reads

$$
\frac{\partial}{\partial t} c(\mathbf{r}, t)=\frac{D}{2} \nabla^{2}\left[2 c(1-2 c)(1-c)-\nabla^{2} c\right]
$$

for the order parameter $c$, and diffusion parameter set to unity in all that follows. The two thermodynamically stable homogeneous states correspond to $c=1$ (hereafter referred to as a liquid phase) and $c=0$ (corresponding to the vapor); the third homogeneous solution, $c=1 / 2$, is unstable. The surface tension associated to the interface between the liquid and gas phases is given by

$$
\sigma=\int d x\left(\frac{d c(x)}{d x}\right)^{2}=\frac{\sqrt{2}}{6},
$$

where $c(x)$ is a one-dimensional (1D) solution of Eq. (1) satisfying the boundary conditions $c(-\infty)=0, c(\infty)=1$, i.e., $c(x)=\frac{1}{2}[1-\tanh (x / \sqrt{2})]$. In all that follows, Eq. (1) is solved numerically using an Euler algorithm. Keeping in mind the arrangement of Fig. 1, we perform our calculations for the two-dimensional case, although a three-dimensional arrangement is also possible. The situation shown in Fig. 1 corresponds to a broad cuvette of size $128 \times 128$ lattice units, with periodic boundary conditions along the horizontal edges (solid lines) and absorbing boundary conditions along the vertical ones (dashed lines). In another (simpler) calculation, a discrete grid of $128 \times 32$ is used (narrow cuvette, leading to a quasi-one-dimensional situation), with periodic boundary conditions applied on the longer sides, reflecting the boundary condition on the right-hand-side shorter edge and with the absorbing boundary condition on the opposite one.

Since the physical mechanisms of the evaporation process are the same in both cases, we concentrate on what follows in the quasi-1D case. In Fig. 2 we show the time evolution of the concentration $c$ measured along the longer symmetry axis of a system initially prepared as a periodic array of $N$ $=8$ equal droplets of radius 5. Just like in Fig. 1, the calculation shows that the droplets do not disappear consecutively according to their further distance from the absorbing boundary but that, for this particular configuration, the actual sequence of the numbers of droplets to evaporate is $1,2,4,7$, $3, \ldots$. This behavior can be attributed to a sort of competitive evaporation between droplets. The time evolution of the radii of the corresponding droplets, measured as the distance from the center of the droplet to the points at which $c(r, t)$ $=0.5$, is shown in Fig. 3. In order to check that the competition between droplets, mediated by matter redistribution among them, is really caused by the effect of the absorbing boundary, we have made a parallel calculation of the evolution of the same initial distribution of droplets but this time with all boundary conditions being periodic. In this case, the droplets evolve towards their equilibrium shape without any considerable redistribution.

We turn now to a qualitative discussion of the effect found. The evolution of the droplet's radius strongly depends on the concentration of the surrounding gas phase. For each

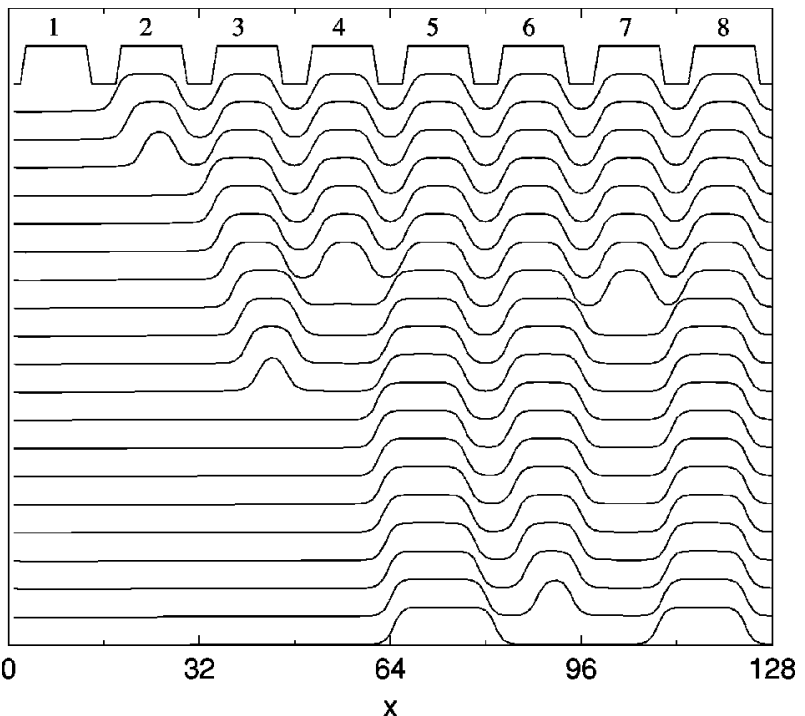

FIG. 2. Time evolution of the concentration profile in an initially periodic, quasi-one-dimensional array of eight droplets (see text for details). The first curve corresponds to $t=0$ (initial condition) and the time increment between two subsequent snapshots is set to $\delta t=500$.

such concentration a critical radius exists, so that the larger droplets grow while the smaller ones dissolve [5]. As Fig. 3 shows, the evaporation of a (subcritical) droplet is a rather fast process, producing a considerable amount of matter diffusing in a bulk gas phase. The matter set free during such an elementary process diffuses not only in the direction of the absorbing boundary, but also in the opposite direction. This matter can be absorbed by the neighboring droplet whose radius then starts to grow. The radius of this droplet can thus get larger than the radius of its next neighbor to the right, so that the droplet of the larger radius gets transiently stabilized, and proceeds to grow further now at the cost of its next neighbor to the right, which will in turn be the next droplet to disappear. The overall sequence of the droplets to evaporate depends thus on the geometry of array (radii of droplets and the distances between them) so that the sequence of evapo-

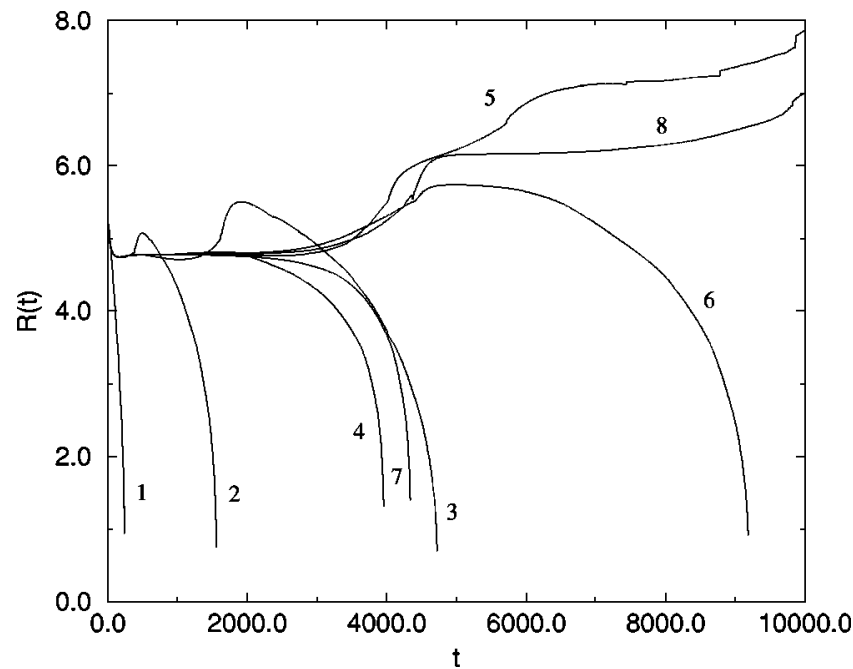

FIG. 3. Time evolution of radii of different droplets, numbered in accordance with Fig. 2. 


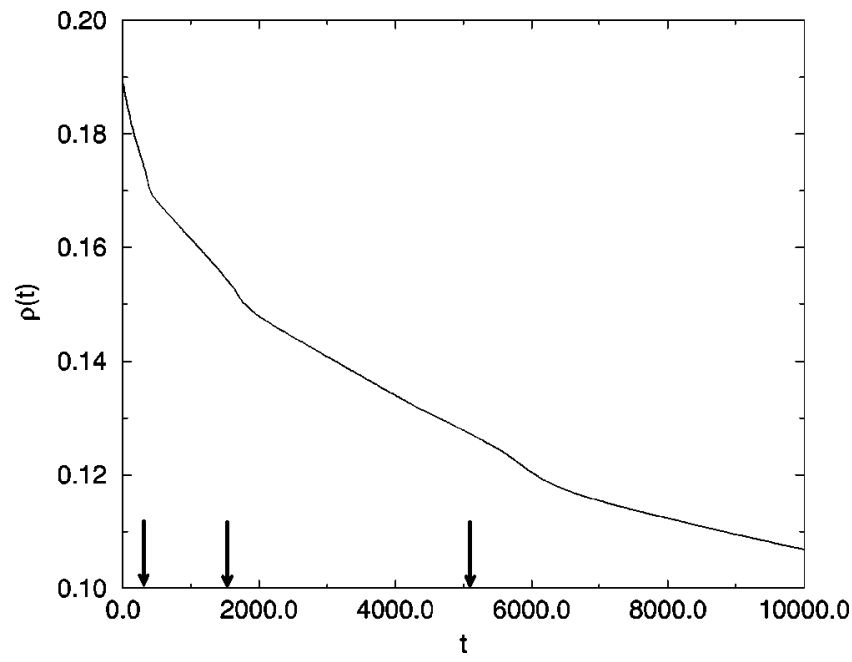

FIG. 4. Time dependence of the mean density of matter in the system. The subsequent linear parts of the curve are controlled by droplets no. 1, 2, 3, and 5. The arrows indicate the time of dissolution of droplets no. 1, 2, and 3 in Fig. 3.

ration can be found only by explicitly solving Eq. (1) under prescribed geometrical conditions. It is also worth discussing the time dependence of the overall amount of matter $\rho$ in the system. The $\rho(t)$ dependence obtained numerically by integrating $c(x, t)$ over the system's volume is shown in Fig. 4. The dependence there depicted can qualitatively be described by a sequence of smooth, practically linear segments connected by more or less pronounced crossover regions, related to the times of evaporation of the leftmost droplet present in the system. The dissolution of internal droplets has no significant effect on the $\rho(t)$ dependence.

We proceed now to show that indeed the overall rate of mass decay in the system is mostly controlled by the leftmost droplet, because its evaporation determines the concentration profile (and therefore the mass flux) at the absorbing boundary. This profile admits a simple analytical treatment. Note that the concentration $c$ in the bulk regions outside of the droplets is small, so that Eq. (1) can be linearized in this region leading in the lowest-order approximation to a simple diffusion equation for $c,(\partial / \partial t) c=\nabla^{2} c$. In a quasistatic approximation, the concentration profile established between the boundary and the leftmost droplet is thus given by

$$
c(x)=c_{R} \frac{x}{l}
$$

where $c_{R}$ is the concentration in the gas phase in the vicinity of the droplet's boundary and $l$ is the distance from the droplet's boundary to the absorbing edge. We recall here that $c_{R} \propto 1 / R$ (with $R$ being the droplet's radius) due to the effects of the surface tension (Laplacian pressure). The diffusive flux to the boundary is then given by

$$
J_{x}(x=0)=-\left.D \frac{d c(x)}{d x}\right|_{x=0}
$$

so that the slope of the $\rho(t)$ dependence is

$$
\frac{d \rho}{d t} \propto-\frac{D}{R l} .
$$

The results of Fig. 3 suggest that the controlling droplets keep both their radius and their position almost constant during considerable time, so that a piecewise-linear time dependence appears. The results of our calculations confirm the dependence (5). The same behavior and interpretation apply to the two-dimensional arrays of droplets of Fig. 1.

Moreover we have also found that the overall structural features of the process are stable against small fluctuations in both droplet positions and sizes, while in the case of strongly disordered arrays these effects may show up in the form of a complex time evolution of the averaged concentration profile. This question is presently under study.

We conclude by summarizing our findings. We consider the patterns and kinetics emerging during the evaporation of periodic arrays of initially equal droplets in two-dimensional systems with open (absorbing) boundaries. Based on the numerical solution of the Cahn-Hilliard equation we show that in this case a reversion of the sequence of the droplets to evaporate next is observed: the droplets which are further from the boundaries may evaporate earlier than those in each boundary's vicinity. This effect is explained by taking into account the complex existing interplay between the matter disappearance through the boundaries and its redistribution between the droplets. Thus the matter set free during the evaporation of the droplets closest to the boundaries is partly absorbed by their neighbors in the interior of the system and stabilizes them. We also show that the overall time dependence of the amount of matter in the system shows piecewise-linear behavior due to the fact that the matter loss is mostly governed by the evaporation of the outermost droplets, nearest to the boundaries. Such an effect of matter redistribution could also be of major importance when considering chemical processes in systems showing phase separation.

\section{ACKNOWLEDGMENTS}

This research was supported by an ACCIONES INTEGRADAS-DAAD grant, the DFG, the Dirección General de Investigación Científica y Técnica (Spain) under Project Nos. PB96-0241 and PB96-1001, and by the Comissionat per Universitats i Recerca de la Generalitat de Catalunya. We also acknowledge computing support from Fundació Catalana per a la Recerca.
[1] S. J. Langer, in Solids Far From Equilibrium, edited by C. Godreche (Cambridge University Press, Cambridge, England, 1992).

[2] J. D. Gunton, M. San Miguel, and P. Sahni, in Phase Transi- tions and Critical Phenomena, edited by C. Domb and J. L. Lebowitz (Academic, New York, 1983), Vol. 8.

[3] I. M. Lifshitz and V. V. Slyozov, J. Phys. Chem. Solids 19, 35 (1961). 
[4] P. C. Hohenberg and B. I. Halperin, Rev. Mod. Phys. 49, 435 (1977).

[5] E. M. Lifshitz and L. P. Pitaevskii, Course on Theoretical Physics, Physical Kinetics Vol. 10 (Pergamon, Oxford, 1981).

[6] J. W. Cahn and J. E. Hilliard, J. Chem. Phys. 28, 258 (1958);
31, 688 (1959).

[7] A. M. Lacasta, A. Hernández-Machado, and J. M. Sancho, Phys. Rev. B 48, 9418 (1993).

[8] A. M. Lacasta, J. M. Sancho, and C. Yeung, Europhys. Lett. 27, 291 (1994). 